\title{
STRESS COMPENSATION BY GaP MONOLAYERS FOR STACKED InAs/GaAs QUANTUM DOTS SOLAR CELLS
}

\author{
D. Alonso-Álvarez ${ }^{1}$, A. G. Taboada ${ }^{1}$, Y. González ${ }^{1}$, J. M. Ripalda ${ }^{1}$, B. Alén ${ }^{1}$, L. González ${ }^{1}$, Jorge M. García ${ }^{1}$, F. Briones ${ }^{1}$, \\ A. Martí ${ }^{2}$, A. Luque ${ }^{2}$, A. M. Sánchez ${ }^{3}$ and S. I. Molina ${ }^{3}$ \\ ${ }^{1}$ Instituto de Microelectrónica de Madrid, CNM (CSIC). c/lsaac Newton 8, PTM, 28760 Tres Cantos, Madrid, Spain \\ ${ }^{2}$ Instituto de Energía Solar, IES-UPM.Ciudad Universitaria,28040, Madrid, Spain \\ ${ }^{3}$ Departamento de Ciencia de los Materiales e Ing. Metalúrgica y Q. I. Universidad de Cádiz, Campus Universitario de \\ Puerto Real, 11510 Puerto Real, Cádiz, Spain
}

\begin{abstract}
In this work we report the stacking of 10 and 50 InAs quantum dots layers using 2 monolayers of $\mathrm{GaP}$ for stress compensation and a stack period of $18 \mathrm{~nm}$ on GaAs (001) substrates. Very good structural and optical quality is found in both samples. Vertical alignment of the dots is observed by transmission electron microscopy suggesting the existence of residual stress around them. Photocurrent measurements show light absorption up to $1.2 \mu \mathrm{m}$ in the nanostructures together with a reduction in the blue response of the device. As a result of the phosphorus incorporation in the barriers, a very high thermal activation energy ( $431 \mathrm{meV})$ has also been obtained for the quantum dot emission.
\end{abstract}

\section{INTRODUCTION}

\section{General}

In recent years, III-V semiconductor quantum dots (QD) have received great interest due to their advantages and unique properties as zero dimensional systems. Most of their applications require stacking multiple QDs layers to increase the gain of the devices. However, this strategy is well known to also increase the accumulated stress in the material during growth generating dislocations and nonradiative recombination centres [1]. The introduction of stress compensation (SC) layers to reduce the accumulated stress is a promising way for improving the material quality and the efficiency of devices based on QDs [2,3]. For the InAs/GaAs QDs system this approach consists of introducing between two consecutive InAs QDs layers a material with smaller lattice parameter than the GaAs matrix one. This material introduces a tensile stress which opposes to the compressive stress generated by the $\ln A s$ quantum dots and prevents the accumulation of excessive stress along the sample, responsible for the appearance of dislocations and other defects. Several materials have been used for SC of the InAs/GaAs QDs system such as InGaAs [4], GaP [2], GaAsP [5] or GaNAs [6]. In this work we report the stacking of 50 InAs QDs layers using $2 \mathrm{GaP}$ monolayers (ML) for SC and a stack period of only $18 \mathrm{~nm}$ on GaAs (001) substrates. We chose a "digital" stress compensation method by the introduction of single monolayers of GaP instead of a GaAsP alloy due to the difficulty to control the phosphorus to arsenic incorporation ratio during growth of the stacked QDs structure.

\section{Sample design and growth}

We have made three assumptions to estimate the amount of GaP monolayers necessary to compensate the stress introduced by the InAs QDs: a) the InAs and GaP layers grow pseudomorphically on $\mathrm{GaAs}$, b) the layer thicknesses do not depend on the in plane strain, and c) there is only biaxial stress during growth. The third condition, is not strictly valid for QDs but simplifies the calculation and will serve as a first aproximation. With these premises and using the equations found in EkinsDaukes et al [7] we obtain:

$$
\begin{gathered}
\frac{n_{G a P}}{n_{I n A s}}=-\frac{A_{I n A s} \varepsilon_{I n A s}}{A_{G a P} \varepsilon_{G a P}} \cong 0.9 \\
A=C_{11}+C_{12}-\frac{2 C_{12}^{2}}{C_{11}}
\end{gathered}
$$

where $n_{\mathrm{GaP}}, \varepsilon_{\mathrm{GaP}}, \mathrm{n}_{\operatorname{lnAs}}$ and $\varepsilon_{\text {InAs }}$ are the number of monolayers and the lattice mismatch of $\mathrm{GaP}$ and InAs respectively and $C$ the elastic stiffness coefficients. In our samples we use $2 \mathrm{ML}$ of InAs to grow the QDs so equation (1) gives a GaP thikness of 1.8 ML. However, we round up this quantity to $2 \mathrm{ML}$ in order to have an entire number of $\mathrm{GaP}$ monolayers which is easier to control during growth.

All samples were grown on GaAs (001) substrates using a solid source molecular beam epitaxy (MBE) system. To test the SC effect, two samples with ten QDs layers each were grown with and without GaP stress compensating layers. In the first sample (sample A), two $\mathrm{ML}$ of $\mathrm{GaP}$ are introduced in the GaAs spacer layer by each QDs layer grown, $1 \mathrm{ML}$ of GaP placed $1.53 \mathrm{~nm}$ below the QDs and a second GaP ML, placed $12.6 \mathrm{~nm}$ above them. The second sample (sample B) was grown without $\mathrm{GaP}$ monolayers as a reference, .The spacer layer thickness between QD was $18 \mathrm{~nm}$ in both samples. This sequence was repeated ten times and buried under $72 \mathrm{~nm}$ 
thick GaAs barrier An AFM layer was grown on top to study the surface quality. All layers were grown at a substrate temperature (Ts) of $530^{\circ} \mathrm{C}$, a Beam Equivalent Pressure $(B E P)$ of $A s_{4}=2 \times 10^{-6}$ Torr was employed for the growth of $\mathrm{GaAs}$ and InAs layers and a BEP $\left(\mathrm{P}_{2}\right)=2.3 \times 10^{-7}$ Torr was used for the growth of GaP layers. For QDs formation $2 \mathrm{ML}$ of $\operatorname{InAs}$ were deposited at $0.021 \mathrm{ML} / \mathrm{s}$. Before and after the GaP monolayers growth all shutter cells were closed during 25 and 30 s respectively to pump out the excess of the group $\mathrm{V}$ element for avoiding the formation of the undesirable ternary alloys.

A third sample was grown to test the benefits of the SC method in a real device. We fabricated an intermediate band solar cell structure [8] containing 50 InAs QDs layers with GaP stress compensation (grown following the previous sequence). Standard optical lithography and wet etching techniques were used to define mesas and metal ohmic contacts.

Table 1: XRD data obtained from the (004) symmetric reflection, including the distance between the GaAs substrate peak and the zero order peak, $\Delta \theta$, the average perpendicular strain, $\left\langle\varepsilon_{\perp}\right\rangle$, the relative strain reduction with respect to the reference sample (sample $B$ ) and the stress compensation degree.

\begin{tabular}{cccc}
\hline \hline & Sample B & Sample A & Simulation \\
\hline \hline$\Delta \theta$ (arcs) & 581 & 491 & 309 \\
$<\varepsilon_{\perp}>$ & 0.004368 & 0.003679 & 0.002403 \\
$\begin{array}{c}\text { Rel. strain } \\
\text { reduction (\%) }\end{array}$ & - & 19 & 45 \\
SC degree (\%) & 0 & 42 & 100 \\
\hline \hline
\end{tabular}
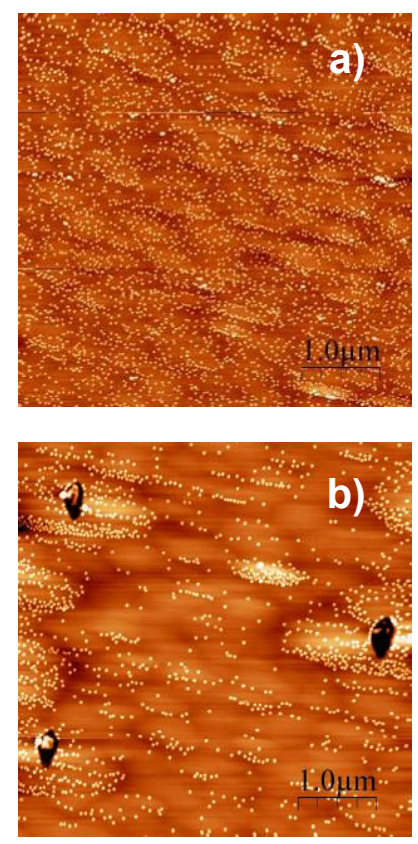

Fig. 1: $5 \times 5 \mu \mathrm{m}$ AFM images of samples a) with and b) without SC.

\section{TEN LAYERS SAMPLES}

\section{Structural characterization}

Sample growth was monitored by reflection high energy electron diffraction (RHEED). The RHEED pattern 2D-3D transition related with the QDs formation was not observed in sample $B$ beyond the fourth layer, indicating excessive defect formation due to the accumulated strain. $\mathrm{Ng}$ et al [1] reported the formation of large "volcano-like" defects in this system that will concentrate most of the In adatoms and prevent the formation of normal size QDs. In sample $A$ we observed the $3 \mathrm{D}$ transition on the ten layers with a nucleation time constant from the third layer, suggesting well controlled In interdifussion during growth and proper stress compensation.

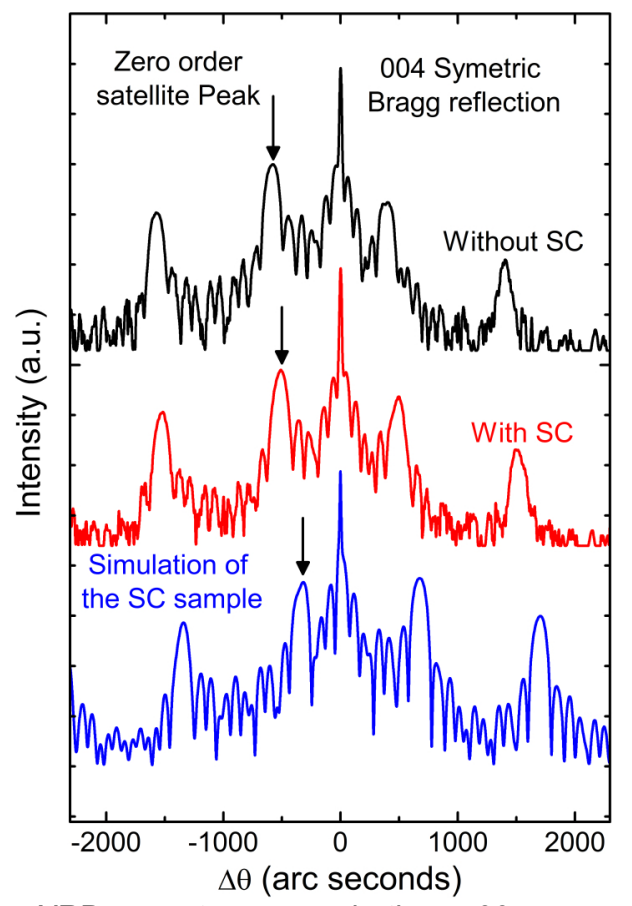

Fig. 2: XRD spectra around the <004> symmetric reflection. Arrows indicate the zero order satellite peak of each spectrum.

This result is supported by atomic force microscopy (AFM) measurements performed on the top layer which also indicates an improved structural quality of the sample A (figure 1a), with very uniform QDs size $(9 \mathrm{~nm}$ high, $50 \mathrm{~nm}$ in diameter) and distribution $\left(1.7 \times 10^{10} \mathrm{~cm}^{-2}\right)$. Figure $1 \mathrm{~b}$ corresponds to sample $B$ and shows large surface undulation, QDs clusters and dark spots that would be associated to the "volcano-like" structures extending to the surface [1].

$X$-ray diffraction (XRD) measurements around the (004) symmetric reflection in $\omega / 2 \theta$ geometry are used to quantitatively analyze the SC effect on the strain accumulation and lattice distortion of samples A and B. The experimental XRD spectra are shown in figure 2, together with a simulated spectra for the nominal structure 
of sample A. Notice that this sample was designed accordingly to the results obtained from expression (1) in order to obtained a zero stress InAs QDs stacked sample by introduction of $\mathrm{GaP}$ SC layers. The perpendicular average strain can be determined by [4]:

$$
\left\langle\varepsilon_{\perp}\right\rangle=\frac{\sin \theta_{B}}{\sin \left(\theta_{B}+\Delta \theta\right)}-1
$$

where $\theta_{B}$ is the Bragg angle of the GaAs substrate and $\Delta \theta$ is the distance between the GaAs substrate peak and the position of the zero order satellite peak. The results are summarized in Table 1.

A $19 \%$ of relative strain reduction with respect to the reference sample (sample $B$ ) has been achieved as a consequence of the introduction of $\mathrm{GaP}$ stress compensating layers. This reduction does not fulfil the zero stress condition, represented by the simulated spectrum of the nominal structure of sample $A$ and that would lead to a $45 \%$ of relative strain reduction. Moreover, this result indicates that the GaP layer thicknesses may be smaller than their nominal values, suggesting phosphorus incorporation into the sample during growth lower than the expected. In spite of the reduced SC degree obtained (only $42 \%$ ), it was enough to allow the growth of ten QDs layers keeping good structural quality. It is interesting to notice that, as pointed out by Ekins-Daukes et al [7], a perfectly stress compensated sample (simulation of the nominal structure of sample A) does not imply a zero perpendicular average strain, that is, the coincidence of the substrate peak and the zero order satellite peak (figure 2 ). Hence, a relative strain reduction of $100 \%$ should not be expected for a perfect compensated sample and simulations or ad hoc calculations have to be used to predict the maximum value.

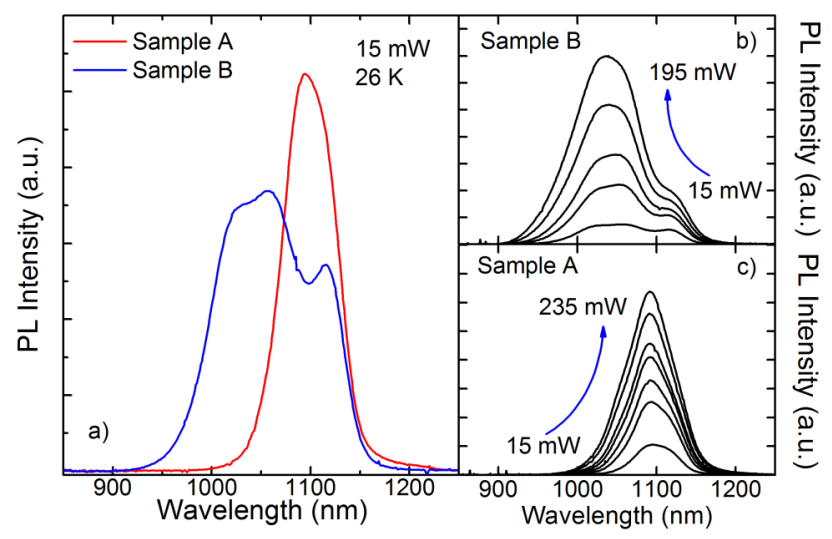

Fig. 3: a) Low temperature PL at $532 \mathrm{~nm}$. Evolution of the PL with excitation power of $b$ ) sample $B$ and $c$ ) sample $A$.
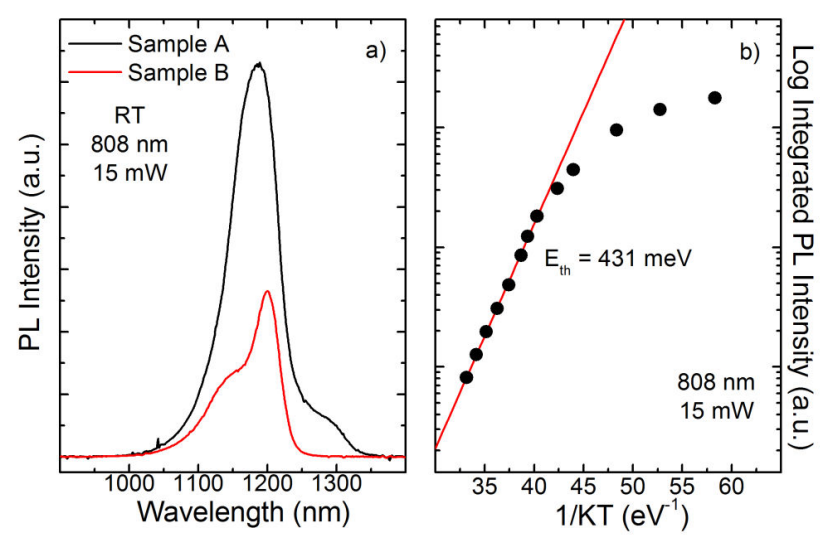

Fig. 4: a) Room temperature PL. b) Temperature evolution of the integrated PL intensity of sample $A$.

\section{Optical properties}

Figure $3 a$ shows the photoluminescence spectra recorded at low temperature of samples $A$ and $B$. Up to three contributions can be clearly observed in sample $B$, at $1.11 \mathrm{eV}, 1.17 \mathrm{eV}$ and $1.21 \mathrm{eV}$, Each of them must be associated to QDs of different average size, as suggested by the evolution with excitation power (figure $3 b$ ). On the other hand, a very good optical quality is observed in sample $A$, with a much narrower peak centered at $1.13 \mathrm{eV}$ (full width at half maximum of $61 \mathrm{meV}$ ). The peak bandwidth and its evolution with the excitation power (figure 3c) suggest the existence of two dinstint QDs families very close in size, given their similar emission energy. These measurements support the improvement of the material quality as a consequence of the introduction of $\mathrm{GaP}$ layers, with almost a complete suppression of the QD size inhomogeneity.

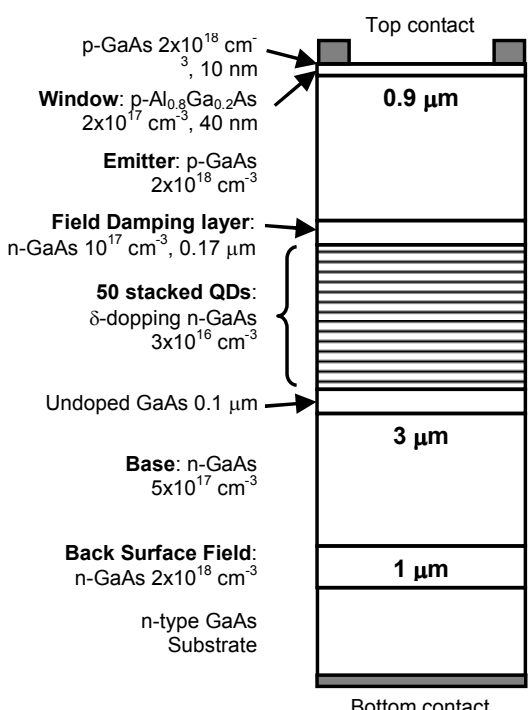

Fig. 5: Structure of the quantum dots solar cell. 
Room temperature photoluminescence (figure 4a) shows that the PL intensity of sample $A$ is increased by a factor of 2.4 compared to sample $\mathrm{B}$. The fitting of the $\mathrm{PL}$ temperature dependence in figure $4 b$ to an Arrhenius-type equation in the high temperature range yields a thermal activation energy for sample A of $431 \mathrm{meV}$, double than that obtained for InAs/GaAs QDs [9]. This enhancement is the consequence of fewer non-recombination centers and a larger confinement of carriers in the nanostructures provided by $\mathrm{P}$ incorporation into the matrix. The exceptional thermal stability obtained suggests this structure also for light emitting applications.

\section{THE QUANTUM DOT SOLAR CELL}

Having tested the stress compensation method in a ten QD layers sample, we fabricated a $\mathrm{GaAs}$ based solar cell with fifty QDs layers in its active region using the described scheme. The goal of this sample is to demonstrate the quantum dot intermediate band solar cell (IBSC) principles as proposed by Marti et al [8]. The structure of the sample can be seen in figure 5 and includes an AIGaAs top layer and a back surface field layer to prevent minority carrier recombination in the top and bottom interfaces respectively. A field damping layer has been inserted between the emitter and the intrinsic region, which contains the dots, to screen the electric field generated by the former and to keep all the QDs layers partly filled with electrons.

\section{Structural characterization}

In situ RHEED measurements not only show the QDs nucleation in the fifty layers of the sample but also indicate excepcional uniformity of the QDs layers. As shown in figure 6 , we obtained an average critical thickness of $1.62 \mathrm{ML}$ with very small distribution broadening. This fact is also important to improve the QD size dispersion through out the fifty layers.

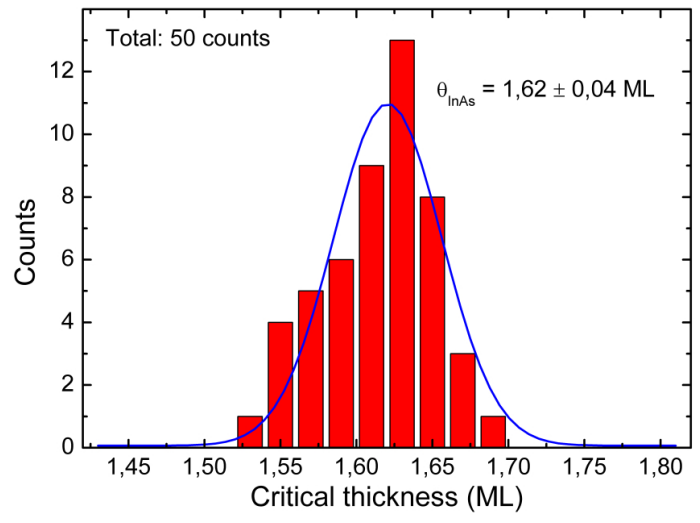

Fig. 6: Distribution of the critical thickness for the 3D transition of the 50 QDs layers.

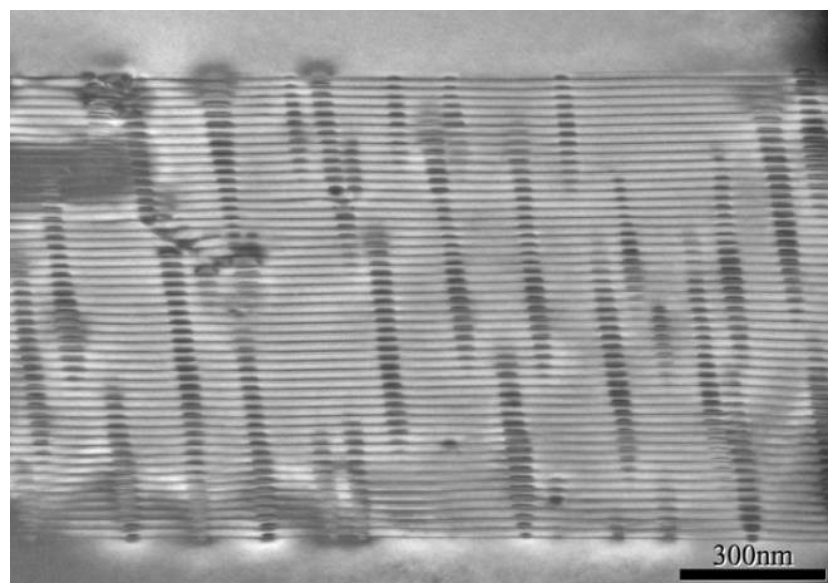

Fig. 7: Cross-section TEM image of the 50 stacked QDs solar cell with SC.

The structural quality of the sample has been tested by means of cross-section transmission electron microscopy (XTEM) (figure 7). As it can be seen, the structure is almost defect free with only some planar defects in the very last few layers. These defects seem to be produced by the interaction of very close or especially large QDs. The image also reveals columnar growth of the QDs along the structure. The direction of the column is not vertical, but forms a angle of $7^{\circ}$ with the growth direction. The stacking behaviour suggests the existence of residual stress around the nanostructures that propagates to the following layer creating sites of preferential nucleation. This behavior has already been observed and predicted by ad-hoc simulations in other nanostructures [10]

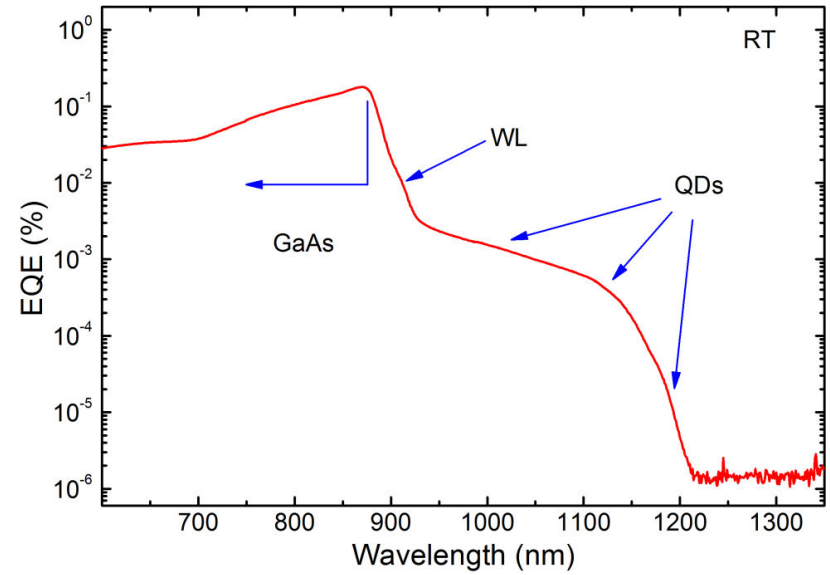

Fig. 8: External quantum efficiency of the quantum dot solar cell. 


\section{Quantum efficiency}

To evaluate the performance of this structure as solar cell, we measure its external quantum efficiency (EQE), defined as [3]:

$$
E Q E=\frac{I_{p h}(\lambda)}{I_{\max }(\lambda)}=\frac{1240 \times I_{p h}(\lambda)}{\lambda P_{i n}(\lambda)}
$$

where $I_{p h}, I_{\max }, P_{\text {in }}$ and $\lambda$ are the photogenerated current (amperes), the maximum possible current (amperes), the incident power (watts) and the incident light wavelength (nanometers) respectively. As it can be seen in figure 8, the QDs solar cell show extended response up to $1.2 \mu \mathrm{m}$ due to absorption in the nanostructures. Several contributions can be distinguished. Signal beyond $950 \mathrm{~nm}$ is associated to transitions of carriers confined in the QDs, including ground and excited states, whereas the hump at $914 \mathrm{~nm}$ is attributed to absorption in the wetting layer (WL). Although the contribution of the nanostructures to the photocurrent is rather small, about three orders of magnitude less than the maximum at the GaAs absorption edge, it should be notice that the IBSC principles require two transitions, one accross the gap to the intermediate band, and other from the intermediate band to the conduction band. In our case, only the former is taking place leaving the later to thermal escape, so the real benefits of including nanostructures in a solar cell can not be evaluated properly in a single wavelength photocurrent experiment. Figure 8 also shows a very poor response of the cell in the short wavelength range. Several effects may contribute to this reduction in the photocurrent. First, the oxidation of the top AIGaAs barrier may have increased, rather than reduced, the recombination rate of electrons at the surface. It is well known that solar cells blue response is greatly affected by recombination of carriers at the surface of the structure [11, 12]. Passivation treatments and thin GalnP or AIGaAs barriers are commonly used to reduce the recombination rate and to keep the electrons away from the surface. However AIGaAs tendency to oxidation, especially in Al rich alloys, may cancel its advantage. Second, defects created in the last few layers, as shown in the TEM image, may have propagated to the surface in the form of dislocations that would trap the photogenerated electrons. Finally, carrier lifetime in InAs QDs is, at least, one order of magnitude smaller than in bulk GaAs. This means that minority carriers that have to cross the junction through the QDs region will tend to recombine instead of being collected in the base and the emitter. In our case, this undesirable recombination effect will be enhanced by two factors: a) the GaP barriers that prevent the free movement of carriers and b) the low electric field in the intrinsic region due to the screening of the field damping layer.

Finally, to further investigate the effect of the nanostructures in the EQE, we performed low temperature photocurrent measurements as a function of the applied bias. As it can be seen in figure 9 , increasing the negative bias also increases the photogenerated current at all wevalengths, as it would be expected due to the extra

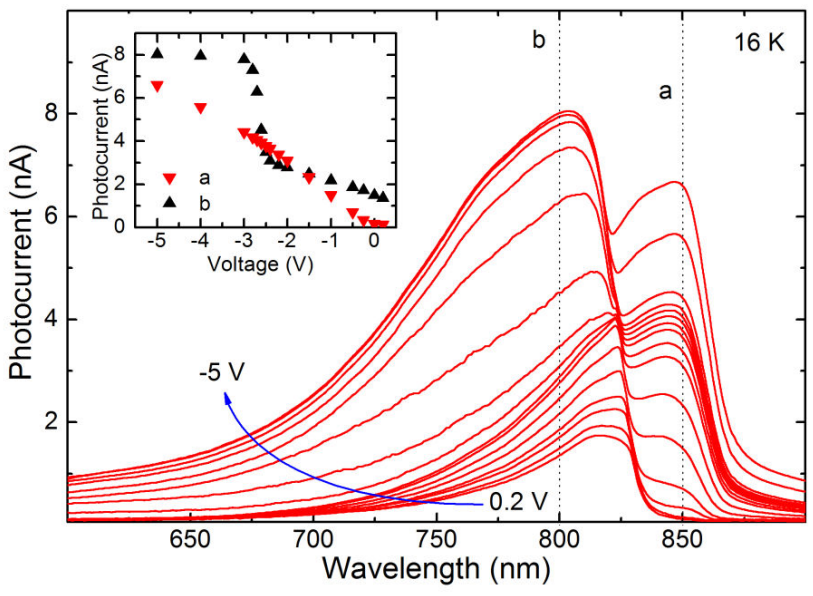

Fig. 9: $P C$ as a function of the reverse bias. The inset shows the PC at wavelengths $a$ and $b$.

electric field. However, the rate at which it increases greatly differs from the low energy to the high energy part of the spectrum. The inset shows the evolution of the photocurrent at two different wavelengths, $850 \mathrm{~nm}$ and $800 \mathrm{~nm}$ marked as $a$ and $b$ respectively in figure 9 . The evolution of wavelength $a$, associated with the WL, is approximately linear with decreasing bias as the confined carriers in more QDs layers are able to tunnel out of the nanostructures, through the $\mathrm{GaP}$ barriers, and being collected. On the other hand, the photocurrent due to absorption of higher energy photons above the GaAs edge, $b$, exhibits a sudden increase at around $-2.5 \mathrm{~V}$, becoming almost constant after that point. We believe that at that voltage carriers begin to have enough energy to overpass the 50 QDs layers, avoiding recombination, and reaching the base.

\section{CONCLUSIONS}

In conclusion, we have studied the effect of "digital" stress compensation with GaP of ten stacked InAs/GaAs layers. Very good structural quality has been found by XRD and $A F M$, and $P L$ measurements have shown thermal activation energy of $431 \mathrm{meV}$. A solar cell including 50 QDs layers with SC has been fabricated with exceptional structural quality, as revealed by the TEM images. The nanostructures have extended the absorption range of the sample up to $1.2 \mu \mathrm{m}$ although an excessive trapping in the QD region has also being found, to the detriment of the short wavelength response of the device. Further work to fully understand and overcome this disadvantage is currently underway. 


\section{ACKNOWLEDGEMENTS}

The authors gratefully acknowledge financial support by the Spanish MEC and CAM through projects 200560M089, S-05050/ENE-0310, TEC-2005-05781-C0301 and -02, Consolider-Ingenio 2010 CSD2006-0004, the Junta de Andalucia (project TEP383, group TEP120) and by the European Commission through the SANDIE Network of Excellence (NMP4-CT-2004-500101).

\section{REFERENCES}

[1] J. T. Ng, U. Bangert, and M. Missous, "Formation and role of defects in stacked large binary InAs/GaAs quantum dot structures", Semiconductor Science and Technology, 22, 2007, pp. 80-85.

[2] J. Tatebayashi, N. Nuntawong, Y. C. Xin, P. S. Wong, S. H. Huang, C. P. Hains, L. F. Lester, and D. L. Huffaker, "Ground-state lasing of stacked InAs/GaAs quantum dots with gap strain- compensation layers grown by metal organic chemical vapor deposition", Applied Physics Letters 88, 2006, pp. 221107.

[3] R. B. Laghumavarapu, M. El-Emawy, N. Nuntawong, A. Moscho, L. F. Lester, and D. L. Huffaker, "Improved device performance of InAs/GaAs quantum dot solar cells with gap strain compensation layers", Applied Physics Letters, 91, 2007, pp. 243115.

[4] N. Nuntawong, S. Birudavolu, CP Hains, S. Huang, H. Xu, and DL Huffaker, "Effect of straincompensation in stacked $1.3 \mu \mathrm{m} \mathrm{InAs} / \mathrm{GaAs}$ quantum dot active regions grown by metalorganic chemical vapor deposition", Applied Physics Letters, 85, 2004, pp. 3050.

[5] N. H. Kim, P. Ramamurthy, L. J. Mawst, T. F. Kuech, P. Modak, T. J. Goodnough, D. V. Forbes, and M. Kanskar, "Characteristics of ingaas quantum dots grown on tensile-strained GaAs1 - xPx", Journal of Applied Physics, 97, 2005 , pp. 093518.

[6] Ryuji Oshima, Takayuki Hashimoto, Hidemi Shigekawa, and Yoshitaka Okada, "Multiple stacking of self-assembled InAs quantum dots embedded by GaNAs strain compensating layers", Journal of Applied Physics, 100, 2006, pp. 083110.

[7] N.J. Ekins-Daukes, K. Kawaguchi, and J. Zhang, "Strain-Balanced Criteria for Multiple Quantum Well Structures and Its Signature in X-ray Rocking Curves", Crystal Growth \& Design, 2, 2002, pp. 287-292.

[8] A. Marti, L. Cuadra, and A. Luque, "Quantum dot intermediate band solar cell", Twenty-Eighth IEEE PVSC, 2000, pp. 940-943.
[9] J. M. Ripalda, D. Alonso-Alvarez, B. Alen, A. G. Taboada, J. M. Garcia, Y. Gonzalez, and L. Gonzalez, "Enhancement of the room temperature luminescence of InAs quantum dots by GaSb capping", Applied Physics Letters, 91, 2007, pp. 012111.

[10] S.I. Molina, T. Ben, D. L. Sales, J. Pizarro, P. L. Galindo, M. Varela, S. J. Pennycook, D. Fuster, Y. Gonzalez, and L. Gonzalez, "Determination of the strain generated in InAs/InP quantum wires: prediction of nucleation sites", Nanotechnology, 17,2006, pp. 56525658.

[11] H.J. Hovel, Semiconductors and Semimetals, vol. 2, New York: Academic Press, 1975.

[12] A. Luque and S. Hegedus, Handbook of Photovoltaic Science and Engineering, Wiley, West Sussex, England, 2003. 